\title{
Relaciones de la Sociedad Chilena de Enfermedades Respiratorias con el Ministerio de Salud
}

\author{
Chilean Society of Respiratory Diseases and Ministry of Health Relationships
}

Las sociedades cientificas de tipo médico, en este caso, la Sociedad Chilena de Enfermedades Respiratorias (SER), tiene como uno de sus objetivos principales contribuir al desarrollo de sus socios en el ámbito profesional, para lo cual organiza y ejecuta actividades cientificas y docentes. Se establece además como un ente coordinador de los temas y materias que los propios socios le proponen, transformándose en un centro de información y comunicación. Desarrolla campos de interés de sus asociados como cursos de Educación Médica, trabajos colaborativos de investigación y actividades de intercambio como pueden ser de tipo técnico o cultural que se consideren aconsejables en el desarrollo de sus socios y de la especialidad médica. Representa además a sus afiliados en aquellas materias que se le solicite su opinión y difunde los conocimientos científicos, técnicos y académicos que interesen a otras sociedades cientificas o a la comunidad nacional e internacional.

Por su parte, el Ministerio de Salud (MINSAL) de nuestro país, declara como misión institucional contribuir a elevar el nivel de la salud de la población, desarrollar armónicamente los sistemas de salud centrados en las personas, fortalecer el control de los factores que puedan afectar la salud y reforzar la gestión de la red nacional de atención.

Entre las funciones y objetivos que el MINSAL declara, hay algunos de ellos que permiten y es aconsejable una directa relación con las sociedades científicas, como son la formulación de planes y programas generales en materia de salud; dictar normas sobre materias técnicas en salud; formular, evaluar y actualizar el Sistema de Acceso Universal con Garantías Explícitas; establecer protocolos de salud, entendiendo como tal, aquellas guías que le permiten entregar instrucciones sobre manejo operativo de problemas de salud determinados. Otro aspecto que relaciona MINSAL con el quehacer de las sociedades cientificas, es que tiene las atribuciones para establecer un sistema de certificación de especialidades y sub especialidades de los prestadores de salud legalmente habilitados para ejercer su profesión.

En estos aspectos la SER ha sido llamada numerosas veces por el MINSAL para entregar su opinión cientifica en aspectos puntuales que tengan importancia en el quehacer nacional, como ocurrió en la última epidemia de Influenza. Otro ejemplo de esta interrelación es el habitual aporte de nuestra sociedad en confecciones de Guías o Normas, como fue en la de Asma Bronquial, Infecciones Respiratorias, Enfermedad Pulmonar Obstructiva Crónica por nombrar a algunas de ellas.

Otra forma muy importante de la relación MINSAL-SER es nuestra activa participación en la Asociación de Sociedades Cientificas Médicas de Chile, ASOCIMED, donde nuestra opinión está siempre presente y es respetada y considerada tanto por otras sociedades cientificas como por el MINSAL

Un aspecto poco desarrollado aún, es nuestro eventual aporte en el Fondo Nacional de Investigación y Desarrollo en Salud (FONIS). Debemos recordar que este organismo fue creado por el Ministerio de Salud y la Comisión Nacional de Investigación Científica y Tecnológica (CONICYT). Su declaración de principios fue disponer de una iniciativa para promover la investigación y desarrollo de temas relevantes para la toma de decisiones en salud pública. A la fecha, nuestros socios participan en forma individual, pero la SER podría diseñar estrategias para convocar a sus miembros a formar grupos de trabajo que generaran proyectos FONIS que resuelvan preguntas o problemas que sean considerados como de alta relevancia en el desarrollo de la especialidad en temas de salud pública. 
Las sociedades cientificas en el día de hoy, deben adquirir un papel activo en el asesoramiento, constituirse en organismos validados de expresión de los intereses de los investigadores y expresar sus demandas. En esta relación con el MINSAL, debemos además participar en solucionar las controversias técnicas científicas que puedan surgir entre MINSAL y SER que disminuyan la confianza del público, ser capaces de identificar a nuestros mejores especialistas e investigadores para cumplir esta labor en temas específicos y facilitar la relación entre la ciencia y la sociedad.

La alianza entre el MINSAL y las sociedades cientificas como la SER, permite aunar esfuerzos y potenciar las capacidades de ambos en lograr mejores documentos y soluciones más acordes a la realidad y necesidad de nuestra población. De este esfuerzo común, los grandes ganadores serán la comunidad cientifica al obtener información de mayor calidad para su práctica habitual y la comunidad de usuarios, que verán cómo las soluciones que les impactan directamente, tienen un fundamento cientifico que les permitirá mejorar su calidad de vida.

\section{Dr. Mario Calvo G.}

Presidente,

Sociedad Chilena de Enfermedades Respiratorias

Email:mcalvo@uach.cl 